\title{
Editorial
}

\section{Exact and Approximate Solutions for Nonlinear PDEs}

\author{
Baojian Hong, ${ }^{1}$ Dianchen Lu, ${ }^{2}$ Chaudry Masood Khalique, ${ }^{3}$ \\ Alvaro H. Salas, ${ }^{4}$ and Robert A. Van Gorder ${ }^{5}$ \\ ${ }^{1}$ Department of Basic Courses, Nanjing Institute of Technology, Nanjing 211167, China \\ ${ }^{2}$ Faculty of Science, Jiangsu University, Zhenjiang, Jiangsu 212013, China \\ ${ }^{3}$ Department of Mathematical Sciences, International Institute for Symmetry Analysis and Mathematical Modelling, \\ North-West University, Mafikeng Campus, Private Bag X 2046, Mmabatho 2735, South Africa \\ ${ }^{4}$ Department of Mathematics, University of Caldas, Manizales 275, Colombia \\ ${ }^{5}$ Department of Mathematics, University of Central Florida, Orlando, FL 32816-1364, USA
}

Correspondence should be addressed to Baojian Hong; hbj@njit.edu.cn

Received 24 April 2014; Accepted 24 April 2014; Published 7 May 2014

Copyright (C) 2014 Baojian Hong et al. This is an open access article distributed under the Creative Commons Attribution License, which permits unrestricted use, distribution, and reproduction in any medium, provided the original work is properly cited.

In the last few decades, due to the wide applications of nonlinear partial differential equations (NPDEs) in nonlinear science [1], the process of looking for exact or approximate solutions of the NPDEs has played an important and significant role in research of mathematicians, physicists, and engineers [2]. Solutions of NPDEs are useful in the study of the dynamics of nonlinear phenomena such as nonlinear waves in hydrodynamics [3], atmospheric dynamics [4], plasma physics [5], solid state physics [6], and optical fibers [7], and thus they may give more insight into the physical aspects of such problems. Up to now, many powerful methods for obtaining exact or approximate solutions of NPDEs have been presented, such as homotopy perturbation method [8], nonperturbative methods [9], homogeneous balance method [10], Darboux transformation method [11], extended tanhfunction method [12], generalized Jacobi elliptic functions expansion method [13], improved general mapping deformation method [14], general algebraic methods [15], and many other methods [16-18].

The purpose of this special issue is to extend several relatively new approaches and theories on searching for the exact and approximate solutions of NPDEs and to see the latest developments in applications of these methods. The response to this special issue was beyond our expectation. We received thirty-nine submissions in total, and, based on valuable review reports, sixteen original high-quality peerreviewed research articles have been accepted for inclusion within this special issue. These papers contain a variety of topics and approaches, including the variational iteration method, the spectral homotopy analysis method, the homotopy perturbation method, the first integral method, the generalized tanh-function expansion method, the Lie symmetry approach, the auxiliary equation method, and the regular perturbation method, along with several interesting applications. These articles contain some new, novel, and innovative techniques and ideas that may stimulate further research on both the theory and the application of NPDEs.

It is certainly impossible to provide in this short editorial a more comprehensive description of all articles published in this special issue. However, the team of the guest editors believes that the results included reflect some recent trends in research and outline new ideas for future studies of exact and approximate solutions for NPDEs and applications thereof.

\section{Acknowledgments}

We would like to express our sincere gratitude to the authors who have submitted papers for consideration in the special issue. We also thank the reviewers of these papers, whose comments were important for us when making the editorial decisions, for their thorough and timely reviews. Many thanks are also given to the editorial board members of this journal, in recognition of their great support and help in making this special issue possible. We hope that this 
special issue will provide some motivation to stimulate future research on the theory and applications of NPDEs.

\section{Baojian Hong \\ Dianchen Lu \\ Chaudry Masood Khalique \\ Alvaro H. Salas \\ Robert A. Van Gorder}

[17] A. H. Salas, "Exact solutions of coupled sine-Gordon equations," Nonlinear Analysis: Real World Applications, vol. 11, no. 5, pp. 3930-3935, 2010.

[18] R. A. van Gorder and K. Vajravelu, "Analytic and numerical solutions to the Lane-Emden equation," Physics Letters A: General, Atomic and Solid State Physics, vol. 372, no. 39, pp. 6060-6065, 2008.

\section{References}

[1] M. J. Ablowitz and P. A. Clarkson, Soliton, Nonlinear Evolution Equations and Inverse Scattering, Cambridge University Press, New York, NY, USA, 1991.

[2] C. H. Gu, Soliton Theory and Its Application, Springer, Berlin, Germany, 1995.

[3] G. L. D. Sidén and D. R. Lynch, "Wave equation hydrodynamics on deforming elements," International Journal for Numerical Methods in Fluids, vol. 8, no. 9, pp. 1071-1093, 1988.

[4] J. E. Hansen, "Exact and approximate solutions for multiple scattering by cloudy and hazy planetary atmospheres," Journal of the Atmospheric Sciences, vol. 26, no. 3, pp. 478-487, 1969.

[5] K. E. Lonngren, "Soliton experiments in plasmas," Plasma Physics, vol. 25, no. 9, pp. 943-982, 1983.

[6] E. Yablonovitch, "Inhibited spontaneous emission in solid-state physics and electronics," Physical Review Letters, vol. 58, no. 20, pp. 2059-2062, 1987 .

[7] A. Kobyakov, M. Sauer, and D. Chowdhury, "Stimulated Brillouin scattering in optical fibers," Advances in Optics and Photonics, vol. 2, no. 1, pp. 1-59, 2010.

[8] J. He, "Homotopy perturbation method for bifurcation of nonlinear problems," International Journal of Nonlinear Sciences and Numerical Simulation, vol. 6, no. 2, pp. 207-208, 2005.

[9] A. Lacour, J. A. Oller, and U.-G. Meißner, "Non-perturbative methods for a chiral effective field theory of finite density nuclear systems," Annals of Physics, vol. 326, no. 2, pp. 241-306, 2011.

[10] A. S. Abdel Rady, E. S. Osman, and M. Khalfallah, "The homogeneous balance method and its application to the BenjaminBona-Mahoney (BBM) equation," Applied Mathematics and Computation, vol. 217, no. 4, pp. 1385-1390, 2010.

[11] H. Hu, X. Tang, S. Lou, and Q. Liu, "Variable separation solutions obtained from Darboux Transformations for the asymmetric Nizhnik-Novikov-Veselov system," Chaos, Solitons and Fractals, vol. 22, no. 2, pp. 327-334, 2004.

[12] H. A. Abdusalam, "On an improved complex tanh-function method," International Journal of Nonlinear Sciences and Numerical Simulation, vol. 6, no. 2, pp. 99-106, 2005.

[13] B. Hong, "New Jacobi elliptic functions solutions for the variable-coefficient MKdV equation," Applied Mathematics and Computation, vol. 215, no. 8, pp. 2908-2913, 2009.

[14] B. Hong and D. Lu, "New Jacobi elliptic function-like solutions for the general KdV equation with variable coefficients," Mathematical and Computer Modelling, vol. 55, no. 3-4, pp. 1594-1600, 2012.

[15] B. Hong and D. Lu, "New exact Jacobi elliptic function solutions for the coupled Schrödinger-Boussinesq equations," Journal of Applied Mathematics, vol. 2013, Article ID 170835, 7 pages, 2013.

[16] C. M. Khalique, "Exact explicit solutions and conservation laws for a coupled Zakharov-Kuznetsov system," Mathematical Problems in Engineering, vol. 2013, Article ID 461327, 5 pages, 2013. 


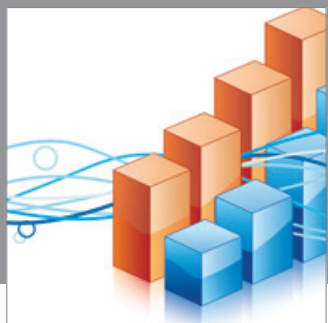

Advances in

Operations Research

mansans

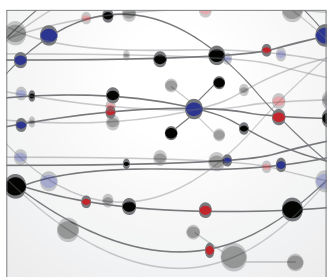

The Scientific World Journal
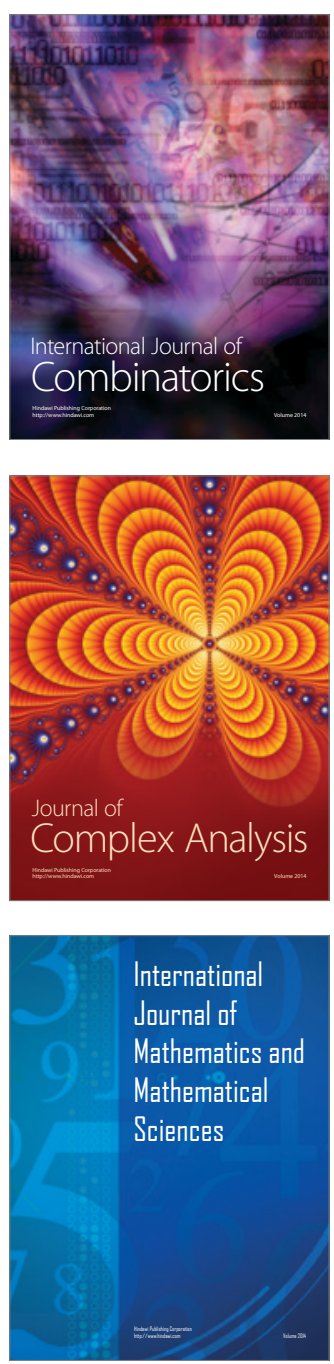
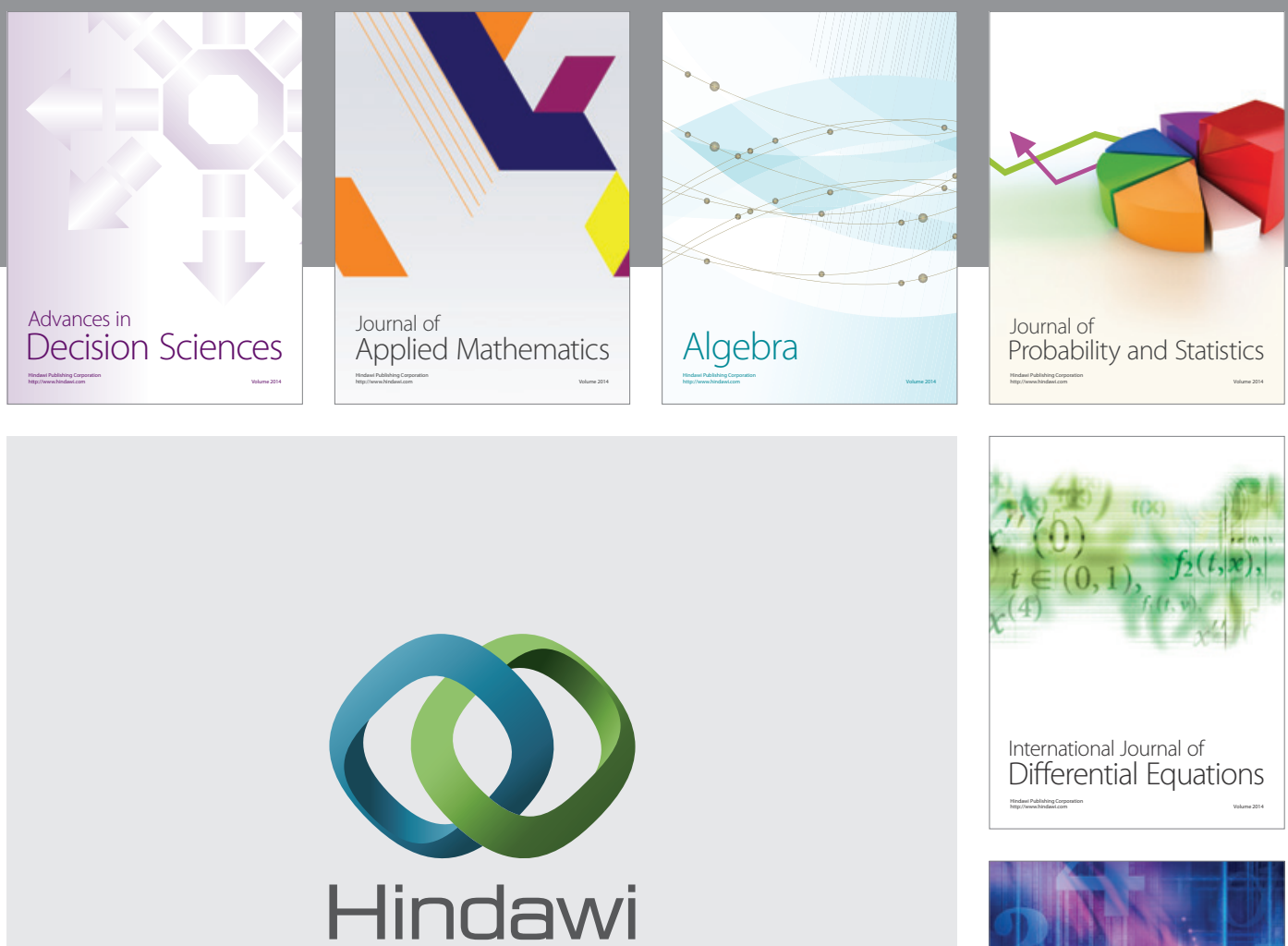

Submit your manuscripts at http://www.hindawi.com
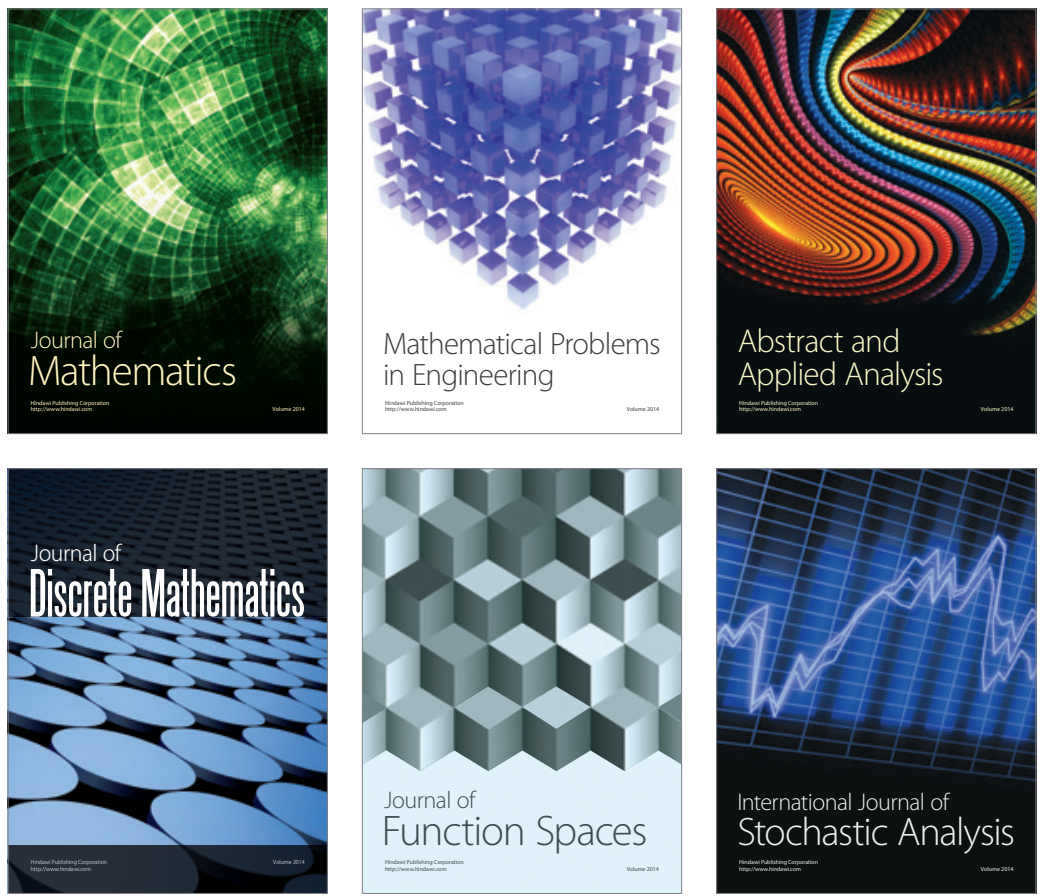

Journal of

Function Spaces

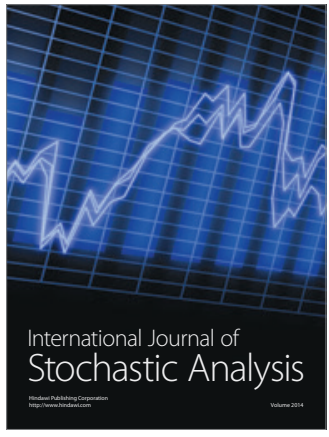

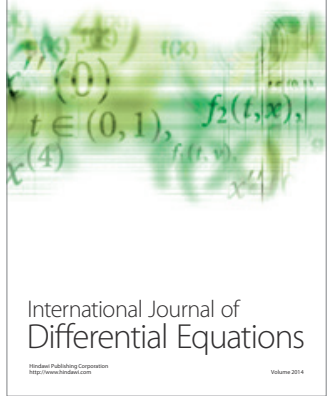
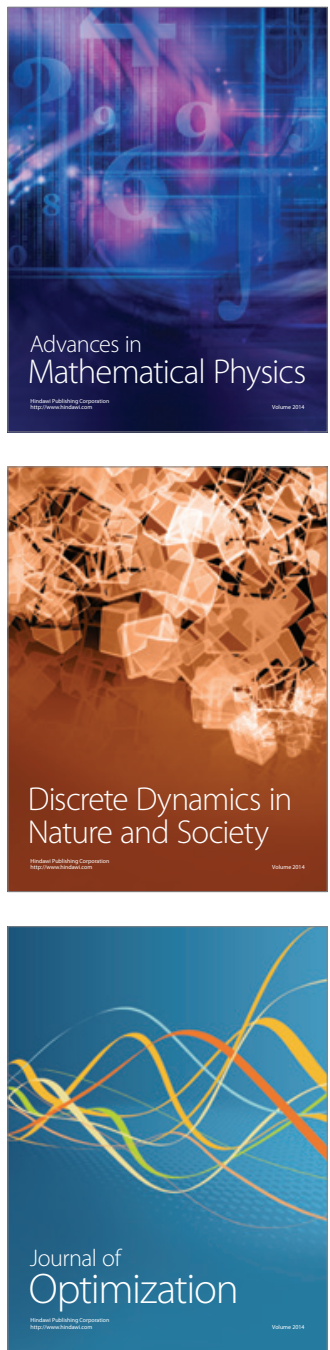